\title{
p53-Dependent PUMA to DRAM antagonistic interplay as a key molecular switch in cell-fate decision in normal/high glucose conditions
}

\author{
Alessia Garufi ${ }^{1}{ }^{12}$, Giuseppa Pistritto ${ }^{3}$, Silvia Baldari ${ }^{1}$, Gabriele Toietta ${ }^{1}$, Mara Cirone ${ }^{4}$ and Gabriella D'Orazi ${ }^{1} 2^{*}$
}

\begin{abstract}
Background: As an important cellular stress sensor phosphoprotein p53 can trigger cell cycle arrest and apoptosis and regulate autophagy. The p53 activity mainly depends on its transactivating function, however, how p53 can select one or another biological outcome is still a matter of profound studies. Our previous findings indicate that switching cancer cells in high glucose $(\mathrm{HG})$ impairs p53 apoptotic function and the transcription of target gene PUMA.

Methods and results: Here we report that, in response to drug adriamycin (ADR) in HG, p53 efficiently induced the expression of DRAM (damage-regulated autophagy modulator), a p53 target gene and a stress-induced regulator of autophagy. We found that ADR treatment of cancer cells in HG increased autophagy, as displayed by greater LC3II accumulation and p62 degradation compared to ADR-treated cells in low glucose. The increased autophagy in HG was in part dependent on p53-induced DRAM; indeed DRAM knockdown with specific siRNA reversed the expression of the autophagic markers in HG. A similar outcome was achieved by inhibiting p53 transcriptional activity with pifithrin-a. DRAM knockdown restored the ADR-induced cell death in HG to the levels obtained in low glucose. A similar outcome was achieved by inhibition of autophagy with cloroquine (CQ) or with silencing of autophagy gene ATG5. DRAM knockdown or inhibition of autophagy were both able to re-induce PUMA transcription in response to ADR, underlining a reciprocal interplay between PUMA to DRAM to unbalance p53 apoptotic activity in HG. Xenograft tumors transplanted in normoglycemic mice displayed growth delay after ADR treatment compared to those transplanted in diabetics mice and such different in vivo response correlated with PUMA to DRAM gene expression.
\end{abstract}

Conclusions: Altogether, these findings suggest that in normal/high glucose condition a mutual unbalance between p53-dependent apoptosis (PUMA) and autophagy (DRAM) gene occurred, modifying the ADR-induced cancer cell death in $\mathrm{HG}$ both in vitro and in vivo.

Keywords: p53, DRAM, PUMA, Autophagy, Hyperglycemia, Diabetes, Chemotherapy, Cancer

\section{Background}

In response to several types of genotoxic stress the p53 oncosuppressor is activated to control, as transcription factor, genes regulating different cellular outcomes such as cell-cycle arrest and apoptosis [1]. In this manner, p53 protects cells from genomic instability leading to

\footnotetext{
* Correspondence: gabriella.dorazi@unich.it

'Department of Research, Advanced Diagnostics, and Technological Innovation, Translational Research Area, Regina Elena National Cancer Institute, Rome, Italy ${ }^{2}$ Department of Medical, Oral and Biotechnological Sciences, Tumor Biology Section, University "G. d'Annunzio", Via de Vestini, 31, 66013 Chieti, Italy

Full list of author information is available at the end of the article
}

tumorigenesis, reduces tumor progression, and activates the apoptotic response of tumor cells to anticancer drugs [2]. Given its key role in restraining tumorigenesis and tumor progression, p53 is frequently mutated in over $50 \%$ of human cancer types and indirectly inactivated in the other $50 \%$, indicating that the presence of a functional p53 pathway is incompatible with neoplastic cell growth [3]. One of the most dramatic effects of p53 activation is the apoptotic clearance of cancer cells [4] which is one of the two ideal goals of anticancer therapy, the other being the stimulation of host tumor-specific 
response, both cooperating in the achievement of clinically relevant effects $[5,6]$. Apoptotic signals can engage two main pathways (the extrinsic and the intrinsic) which are interconnected [7]. P53 is involved in both pathways through the regulation of target genes that encode, for instance, the mitochondrial BH3-domain proteins NOXA and PUMA $[8,9]$. PUMA (p53 upregulated modulator of apoptosis) represents one of the most potent pro-apoptotic BH3-only proteins and a key mediator of p53-dependent apoptosis in response to a wide variety of stress signals including genotoxic stress, double- and single-stranded DNA breaks (i.e., UV, $\gamma$-IR, purine analogues, topoisomerase inhibitors, chemotherapeutic agents, etc.) but also oxidative stress, neurotoxins, changes in microtubule structure, deficiency of growth factors, hypoxia and viral infection [10]. The absence of PUMA has been shown to cause high resistance of cancer cells to apoptosis induced by DNA-damaging agents, such as adriamycin, 5-fluorouracil, cisplatin, etc. [11, 12].

Besides, p53 has been shown to play a critical role in p53 in regulation of autophagy, a catabolic pathway by which eukaryotic cells degrade and recycle macromolecules and organelles, particularly under conditions of nutrient deprivation [13]. This may depend on p53 subcellular localization and/or by p53 transcriptiondependent and -independent activities [14-16]. TP53 may induce autophagy through, for instance, activation of AMPK kinase/mTOR signalling [17] or by transcriptional induction of autophagy genes such as DRAM (damage-regulated autophagy modulator), a lysosomal protein and a stress-induced regulator of autophagy [18]. DRAM has been shown to be not only critical for the ability of p53 to induce autophagy, but also for p53induced apoptotic cell death [19], contributing to the complex mechanisms that regulate whether or not a cell dies in response to p53. Thus, although p53 is involved in the regulation of autophagy, the exact nature of this link remains seemingly controversial [20].

Autophagy, along with apoptosis controls the turnover of organelles, proteins and therefore the cell fate (survival/cell death). Generally autophagy blocks the induction of apoptosis that shuts off the autophagic process and the dialogue between these two pathways influences the normal clearance of dying cells [21]. Thus, autophagy inhibits apoptosis in mammalian cells [22] and facilitates the resistance of tumor cells to anticancer agents [23]. Therefore, knowing the mechanisms that rule the interplay between these two cellular processes (autophagy/apoptosis) has important pathophysiological consequences [24]. Recently we reported that high glucose (HG) condition reduces the tumor cell response to druginduced apoptosis due to impairment of p53 apoptotic function. Mechanistically we found that HG induces homeodomain-interacting protein kinase 2 (HIPK2) protein degradation leading to impairment of HIPK2/ p53 apoptotic axis with inhibition of p53 apoptotic targets PUMA and p53AIP1 $[25,26]$.

In this study, we present evidence that HG condition changes the p53 transcriptional activity from PUMA to DRAM with the consequence of impairment of drug-induced cell death. We also show that p53-induced DRAM sustains HG-triggered autophagy and that either DRAM or autophagy inhibition restore both PUMA transcription and drug-induced cell death in vitro and in vivo.

\section{Methods}

\section{Cell culture and reagents}

In this study colon cancer RKO, HCT116, and HCT116p53 $3^{-1-}$ cells were used. Cells were routinely cultured in DMEM (Dulbecco's Modified Eagle's medium) (Life Technology-Invitrogen, Eggenstein, Germany) containing $1 \mathrm{~g} / \mathrm{L}$ D-glucose (low glucose - LG), supplemented with $10 \%$ heat-inactivated fetal bovine serum (FBS) plus 100 units $/ \mathrm{ml}$ penicillin/streptomycin, and glutamine, in $5 \% \mathrm{CO}_{2}$ humidified incubator at $37{ }^{\circ} \mathrm{C}$. For high glucose (HG) condition, low glucose culture medium was replaced with DMEM containing 4.5 g/L D-glucose (Life Technology-Invitrogen) supplemented with $2 \%$ FBS for $24 \mathrm{~h}$, as previously reported [25-28], before performing other treatments for the indicated times.

To generate a cell line stably expressing GFP-LC3, $3 \times 10^{5}$ RKO cells were cultured into 6-well plates and transfected with $5 \mu \mathrm{g}$ of pEGFP-LC3 expression vector (kindly provided by Moshe Oren, Weizmann Institute of Science, Rehovot, Israel) with Lipofectamine Plus (Invitrogen, Monza, Italy) according to the manufacturer's instructions. Forty-eight hours after transfection cells underwent selection with geneticin G418 (1.5 mg/ml). After 10-14 days, the selected GFP-LC3positive clones were visualized on a Nikon Eclipse Ti-U fluorescence microscope (Nikon).

Chemotherapeutic drug Adriamycin (ADR) (Sigma) was added in culture medium at $2 \mu \mathrm{g} / \mathrm{ml}$ for $16-24 \mathrm{~h}$; the inhibitor of autophagic protein degradation chloroquine (CQ) [29] (Sigma-Aldrich) was added in culture medium at $25 \mu \mathrm{M}$ for $16 \mathrm{~h}$; p53 inhibitor pifithryn- $\alpha$ (PFT- $\alpha)$ [30] (ENZO Life Sciences, Lausen Switzerland) was used at $30 \mu \mathrm{M}$ for $16-24 \mathrm{~h}$, as reported [31].

\section{Viability assay}

For viability assay, subconfluent cells were plated in duplicate in $60 \mathrm{~mm}$ multiwell Petri dishes and $24 \mathrm{~h}$ later culture medium was replaced with HG or low glucose medium, both containing $2 \%$ FBS. The day after, ADR (2 $\mu \mathrm{g} / \mathrm{ml}$ ) were added to cell cultures for $16-24 \mathrm{~h}$. Both floating and adherent cells were collected and cell viability was determined by Trypan blue (Sigma, St. Louis, MO, 
USA) exclusion by direct counting with a haemocytometer. The percentage of cell death, as blue/total cells, was assayed by scoring about 200 cells per well in triplicate.

\section{Cell death/PI staining}

Cell death was quantified by Fluorescence Activated Cell Sorting (FACS) analysis, staining cells with the nonvital dye propidium iodide (PI) (Immunological Sciences, Rome, Italy), following the manufacturer's instruction [32]. Briefly, floating cells were collected by centrifugation and pooled with adherent cells recovered from the plates, fixed in $80 \%$ ethanol and stained in a PBS solution containing PI $(62.5 \mathrm{mg} / \mathrm{ml}$; Sigma-Aldrich), and RNase A (1.125 mg/ml; Sigma-Aldrich). Samples were acquired with a FACScan instrument (Becton Dickinson Europe Holdings SAS - Le Pont De Claix, France) and the percentage of cells in sub-G1 compartment was calculated using ModFit LT software (Becton Dickinson). About 30.000 events were acquired and gated using forward scatter and side scatter to exclude cell debris.

\section{Western blot analysis}

Total cell extracts were prepared by incubation in lysis buffer $(50 \mathrm{mM}$ Tris-HCl, pH 7.5, $150 \mathrm{mM} \mathrm{NaCl}, 5 \mathrm{mM}$ EDTA, pH 8.0, $150 \mathrm{mM} \mathrm{KCl,} 1 \mathrm{mM}$ dithiothreitol, $1 \%$ Nonidet P-40) and a mix of protease and phosphatase inhibitors (Sigma-Aldrich). Protein concentration was then determined using BCA Protein Assay kit (Bio-Rad, Hercules, CA, USA). Samples were then denatured in SDS sample buffer. Total proteins were separated by loading 20-60 $\mu \mathrm{g}$ of total cell lysates on denaturing 620\% SDS-PAGE (Bio-Rad) and transferred to polyvinylidene difluoride (PVDF) (Merck Millipore, Billerica, MA, USA) or nitrocellulose (Bio-Rad) membranes. Unspecific binding sites were blocked by incubating membranes for $1 \mathrm{~h}$ in $0.05 \%$ Tween-20 ( $\mathrm{v} / \mathrm{v}$ in TBS) supplemented with $5 \%$ non-fat powdered milk or bovine serum albumin, followed by overnight incubation with the following primary antibodies: rabbit polyclonal anti-LC3B (SigmaAldrich), mouse monoclonal anti-p62 (SQSTM1, D-3) (Santa Cruz Biotechnology, Dallas, TX, USA); mouse monoclonal anti-poly(ADP-ribose) polymerase (PARP, cleavage site-214/215, Millipore); and mouse monoclonal anti- $\beta$-actin (Calbiochem, San Diego, CA, USA). Primary antibodies were detected with appropriate antiimmunoglobulin-G-horseradish peroxidase secondary antibodies (Bio-Rad). Enzymatic signals were visualized using chemiluminescence (ECL Detection system, Amersham GE Healthcare, Milan, Italy). Images were acquired with the EPSON Expression 10,000 XL scanner (Epson, Long Beach, CA, USA) and densitometry was performed with the ImageJ software (NIH, Bethesda, MD, USA).
RNA extraction and semi-quantitative reverse transcription (RT)-PCR analysis

Cells were harvested in TRIzol Reagent (Life TechnologyInvitrogen) and total RNA was isolated following the manufacturer's instructions, as previously reported [25]. The first strand cDNA was synthesized from $2 \mu \mathrm{g}$ of total RNA with MuLV reverse transcriptase kit (Applied Biosystems, Foster City, CA, USA). Semi-quantitative ReverseTranscribed (RT)-PCR was carried out by using HotMaster Taq polymerase (Eppendorf, Milam Italy) with $2 \mu \mathrm{l}$ cDNA reaction and genes specific oligonucleotides under conditions of linear amplification. PCR products were run on a $2 \%$ agarose gel and visualized with ethidium bromide. The housekeeping 28S gene, used as internal standard, was amplified from the same cDNA reaction mixture. Densitometric analysis was applied to quantify mRNA levels compared to control gene expression.

\section{siRNA interference}

Cells were plated at semiconfluence in $35-\mathrm{mm}$ Petri dishes the day before transfection. Control-siRNA, siDRAM (sc-96,209; Santa Cruz) and siATG5 (Dharmacon, Thermo Scientific, Milan, Italy) were transfected overnight using Lipofectamine Plus reagent (Invitrogen). DRAM silencing was evaluated $48 \mathrm{~h}$ after transfection by RT-PCR analysis.

\section{In vivo tumor growth}

In vivo experiment was performed by using six-week-old CD-1 male nude (nu/nu) mice (Charles River Laboratories). They were housed in specific pathogen-free conditions and fed standard cow pellets and water ad libitum. Studies were performed in accordance with the National Cancer Institute Regina Elena standard guidelines for animal care; all experimental protocol were approved by the Ethical Committee for animal research of the National Cancer Institute Regina Elena in Rome, Italy, and by the Italian Ministry of Health, and performed in accordance with the Italian and European legislation. For diabetes induction the mice were injected intraperitoneally with a single high dose of 160 to $240 \mathrm{mg} / \mathrm{kg}$ streptozotocin (STZ) (Sigma-Aldrich) which is considered to achieve consistently a diabetic state with limited morbidity and mortality [33]. The mice were subsequently hydrated with $1.0 \mathrm{ml}$ normal physiologic saline administrated subcutaneously. Blood was obtained by lancet prick in the tail. Blood glucose concentration was monitored once before and daily after STZ injection until a diabetic state was confirmed by the glucose dehydrogenase method. After mice reached a glucose concentration exceeding $300 \mathrm{mg} / \mathrm{dl}$ (considered diabetic), solid tumors were obtained by injecting i.m. on the flank of each mouse $4 \times 10^{6}$ viable RKO cells suspended in $0.1 \mathrm{ml}$ PBS. The mice were examined every day after 
injection until tumors reached approximately $300 \mathrm{~mm}^{3}$ volume (about 7 days from injection). Mice were then randomized in four groups (6-8 mice/group) and injected with ADR (10 mg/kg body weight) i.p or PBS as control, as follow: 1) normoglycemic/PBS (Mock), 2) normoglycemic/ADR, 3) diabetic/PBS, 4) diabetic/ADR. Tumor dimensions were measured every other day and their volumes were calculated from caliper measurements of two orthogonal diameters ( $x$ and $y$, larger and smaller diameters, respectively) by using the formula, volume $=x y^{2} / 2$. When the ADR effect on tumor growth delay reached statistical significance between normoglycemic and diabetic groups, tumors were harvested and RNA extracted for analysis of PUMA and DRAM expression by RT-PCR.

\section{Statistical analysis}

Each experiment, unless differently specified, was performed at least three times. Results are expressed as values of mean \pm S.D. Statistical significance was determined using Student's $t$-tests for two sample comparisons and one-way ANOVA analysis for three or more sample comparisons.

\section{Results}

High glucose (HG) switched the adriamycin (ADR)-induced p53 transcriptional activity from PUMA to DRAM

We first evaluated whether the glucose amount influenced the p53 transcriptional activity in response to drug. To this aim, HCT116 and RKO cells were cultured in low glucose (LG) and high glucose (HG) media and treated with adriamycin (ADR). The results of RT-PCR analyses of mRNA levels show that PUMA was greatly induced by ADR in LG, as expected [25], while was not induced in HG (Fig. 1a); on the contrary and opposite to PUMA, DRAM was specifically induced by ADR in HG while was not induced in LG (Fig. 1a). To assess if DRAM was specifically induced by $\mathrm{p} 53$ in this setting, as it can be also activated by p73 a p53 family protein [34], we inhibited p53 transcriptional activity with pifithrin- $\alpha$ (PFT- $\alpha)$ [30]. We found that the expression of DRAM, induced by ADR in HG, was efficiently impaired by PFT- $\alpha$ co-treatment (Fig. 1b). Similarly, neither DRAM nor PUMA were induced in HCT116-p53 ${ }^{-/-}$treated with ADR in, respectively, LG and HG (Fig. 1c), demonstrating that both genes were induced by p53 in different glucose culture condition.

HG culture condition increased autophagy in ADR-treated cells in part depending on p53 activity

To evaluate the occurrence of autophagy, RKO cells were stably transfected with GFP-LC3 plasmid and selected as mixed population. Then, cells were cultured in LG and HG media and treated with ADR. Autophagy

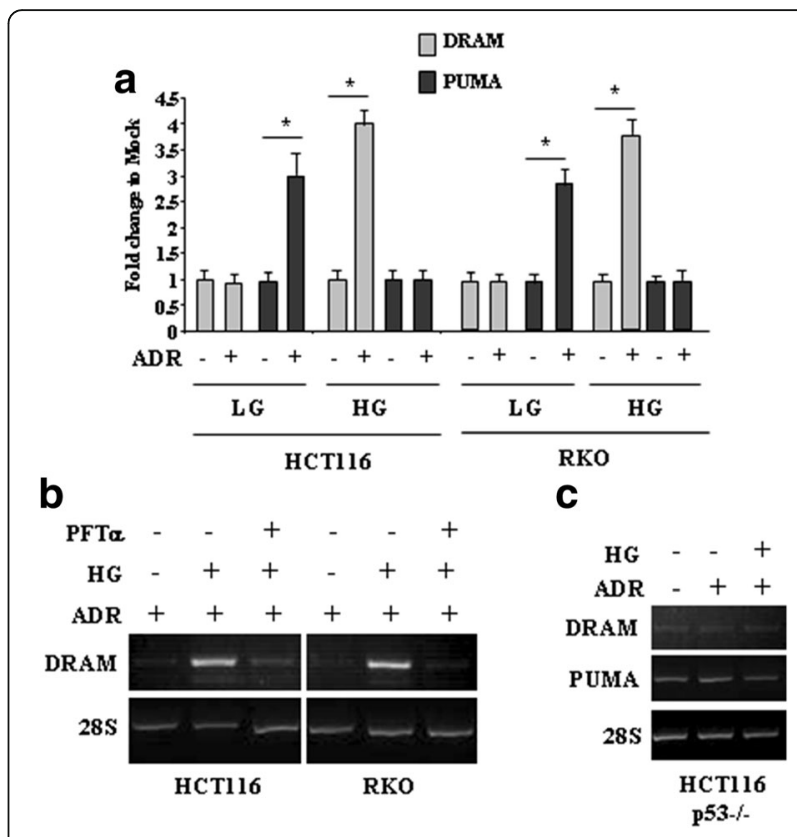

Fig. 1 High glucose (HG) switched the adriamycin (ADR)-induced p53 transcriptional activity from PUMA to DRAM. (a) RKO and HCT1 16 cells were kept in low glucose ( $L G)$ or high glucose $(\mathrm{HG})$ medium for $24 \mathrm{~h}$ and then treated with ADR $(2 \mu \mathrm{g} / \mathrm{ml})$ for $16 \mathrm{~h}$ before being assayed for semi-quantitative RT-PCR analysis of PUMA and DRAM gene expression. 285 was used as a control for efficiency of RNA extraction and transcription. Histograms representing quantification of PUMA or DRAM/28S ratio as assessed by densitometric analysis are shown. Densitometric values were quantified using the ImageJ software and normalized to control. The values of control were set to 1 . The data are presented as means \pm S.D. of three independent experiments. ${ }^{*} P<0.001$. (b) RKO and HCT116 cells were treated with ADR $(2 \mu \mathrm{g} / \mathrm{ml})$ for $16 \mathrm{~h}$ in LG and HG medium, with or without p53 inhibitor pifithrin-a (PFT-a) $(30 \mu \mathrm{M})$ before being assayed for semiquantitative RT-PCR analysis of PUMA and DRAM gene expression. 28S was used as a control for efficiency of RNA extraction and transcription. One representative experiment is shown. (c) HCT116-p53 $3^{-/-}$cells were treated and assayed as in (a)

induction was evaluated by the appearance of GFP-LC3 puncta, indicating autophagosome formation, The cells displaying more than 5 dots/cell were considered undergoing autophagy, and the measurements were performed in the presence of autophagy inhibitor choroquine (CQ), an inhibitor of the lysosomal function and therefore indicative of autophagic flux, as reported [29,35]. We found that the LC3 puncta formation in cells cultured in HG was slightly increased compared to cells cultured in LG and that ADR treatment further increased the LC3 puncta formation in HG compared to the same treatment in LG (Fig. 2a, b). Next Western blot analysis of the expression of LC3 (microtubule-associated protein light chain 3) after conversion from LC3-I to its autophagosome membraneassociated lipidated, activated LC3-II form was performed, in the presence and in the absence of $C Q$. We found that LC3-II conversion, following ADR treatment in LG 

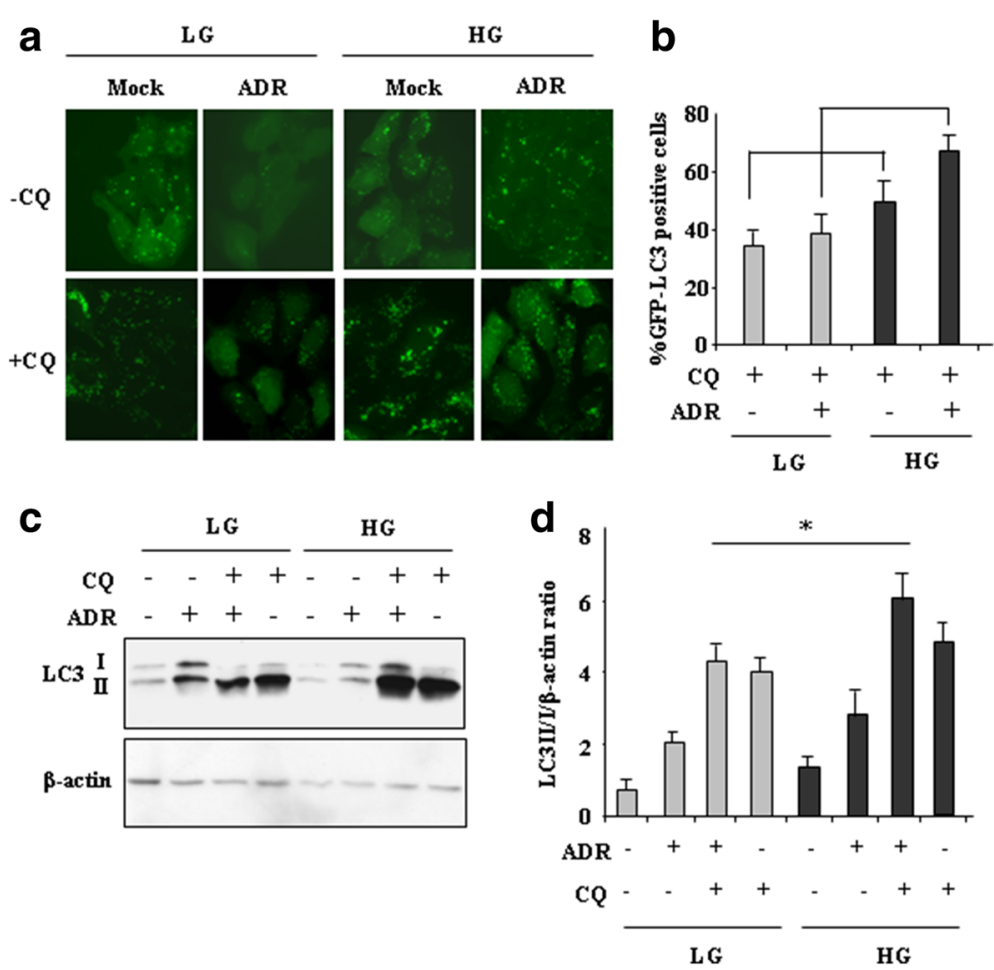

Fig. 2 HG increased autophagy in ADR-treated cells. (a) RKO stably transfected with GFP-LC3 plasmid were kept in low glucose (LG) or high glucose (HG) medium for $24 \mathrm{~h}$ and then treated with ADR $(2 \mu \mathrm{g} / \mathrm{ml})$ for 16 , in the presence or absence of autophagy inhibitor chloroquine (CQ, $25 \mu \mathrm{M})$ for $4 \mathrm{~h}$ before observation to count GFP-LC3 puncta under fluorescence microscopy. Green indicates GFP-LC3. One of 10 representative micrographs is shown. (b) The relative number of GFP-LC3-positive cells was calculated from 10 random fields. The data are presented as the means \pm S.D. from three independent experiments. (c) RKO cells were kept in low glucose (LG) or high glucose (HG) medium for $24 \mathrm{~h}$ and then treated with ADR $(2 \mu \mathrm{g} / \mathrm{ml})$ for $16 \mathrm{~h}$ in the presence or absence of $25 \mu \mathrm{M}$ chloroquine (CQ), and the expression of LC3-1/II was measured by western blot analysis. One representative experiment is shown. $\beta$-actin was used as internal control. (d) Findings as in (c) were assessed by quantitative analysis of $L C 3 \| 1 / 1 / \beta$-actin protein levels and shown as histograms. The data are presented as the means \pm S.D. from three independent experiments. ${ }^{*} P<0.001$

medium, was greatly increased by HG medium (Fig. 2c, d), suggesting increase of autophagy in HG. We then evaluated whether p53 was involved in autophagy increase. To this aim, p53 transcriptional activity was inhibited by using PFT- $\alpha$. As shown in Fig. 3a, the LC3-II conversion following ADR treatment in HG medium was impaired by PFT $\alpha$ co-treatment. Analysis of p62, a bona fide autophagic substrate [35], showed that the reduced levels following ADR treatment in HG were counteracted by PFT $\alpha$ co-treatment (Fig. 3a). The role of DRAM was then assessed by using siRNA interference (Fig. 3b). As shown in Fig. 3c, the degradation of p62 in ADR/HG condition was impaired by DRAM interference. Altogether these findings suggest that HG condition increased the ADR-induced autophagy and that such outcome was in part depending on p53 activity and DRAM expression.

Autophagy inhibition or DRAM silencing restored ADRmediated cell death in HG

Next, we evaluated whether autophagy was involved in reduction of drug-induced cell death in HG. To this purpose cells were treated with ADR in LG and HG, in the presence or absence of autophagy inhibitor CQ. The results show that the ADR-induced cell death in LG, as assessed by PI staining and FACS analysis, was significantly reduced in HG condition; interestingly, blocking autophagy with CQ rescued the ADR-induced cell death in HG approximately to the levels obtained in LG (Fig. 4a, upper panel). CQ alone did not induce cell death (data not shown). In agreement, Western blot analysis show that the expression of the apoptosis marker (cleaved) PARP in response to ADR in LG was reduced by HG and rescued by CQ co-treatment (Fig. 4a, lower panel). To further evaluate the role of autophagy, depletion of ATG5, one of the members of the ATG family necessary for autophagy because of its role in autophagosome elongation [36], was performed with specific siRNA. We found that ATG5 knockdown rescued the cell death inhibited in ADR/HG condition to almost the levels obtained by ADR in LG (Fig. 4b). Next the role of DRAM was assessed in both RKO and HCT116 cells. The results show that the ADR-induced cell death in LG 


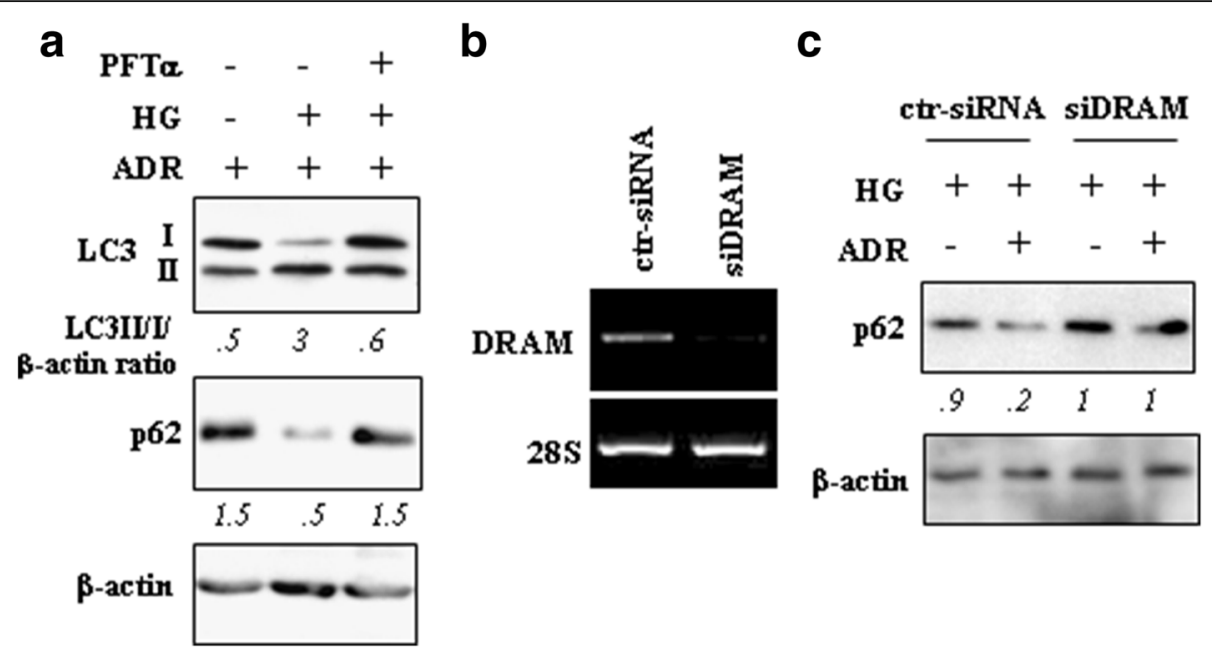

Fig. 3 Increase of autophagy of ADR-treated cells in HG was in part depended on p53 activity. (a) HCT116 cells were treated with ADR (2 $\mu \mathrm{g} / \mathrm{ml}$ ) for $16 \mathrm{~h}$ in LG and HG medium, with or without p53 inhibitor pifithrin-a (PFT-a) (30 $\mu \mathrm{M})$ before being assayed for western blot analysis. Densitometric values of $L C 3 \| / / / \beta$-actin protein levels were quantified using the ImageJ software and normalized to control. One representative experiment is shown. (b) HCT116 cells were transfected with ctr-siRNA and siDRAM and $36 \mathrm{~h}$ after transfection DRAM expression was assessed by RT-PCR analysis. One representative experiment is shown. (c) HCT116 cells, transfected with ctr-siRNA and siDRAM, were kept in HG medium for $24 \mathrm{~h}$ and then treated with ADR ( $2 \mu \mathrm{g} / \mathrm{ml}$ ) for $16 \mathrm{~h}$ before the expression of p62 was measured by western blot analysis. Densitometric values of p62/ $\beta$-actin protein levels were quantified using the ImageJ software and normalized to control and the results are shown under the images. One representative experiment is shown. Anti $\beta$-actin was used as protein loading control

and significantly reduced by ADR treatment in HG condition, was mostly restored by silencing DRAM with siRNA (Fig. 4c). Altogether, these data suggest that autophagy and/or DRAM expression were responsible, at least in part, of reduced cancer cell death in response to ADR in HG condition.

\section{Autophagy inhibition or DRAM silencing restored ADR- mediated PUMA transcription in HG}

Next we evaluated whether blocking autophagy and/or DRAM could influence PUMA transcription in HG. RTPCR analyses of mRNA levels in RKO and HCT116 cells show that PUMA expression was induced by ADR in LG while was not induced in HG (Fig. 5a, compare lane 2 with lane 6), as expected; interestingly, PUMA was induced by ADR in HG only in the presence of CQ (Fig. 5a, compare lane 6 with lane 7), while the ADR-induced PUMA expression in LG was not further increased by CQ co-treatment (Fig. 5a, compare lane 2 with lane 3). As opposite to PUMA, DRAM expression was induced by ADR only in HG, as seen above, but inhibited by $C Q$ co-treatment (data not shown). Interestingly, PUMA was induced by ADR in HG following siRNA silencing of DRAM (Fig. 5b, compare lane 4 with lane 6), as assessed by densitometric analysis (Fig. 5c). Altogether, these findings suggest that the p53 pro-apoptotic function, impaired in HG, could be restored by autophagy inhibition or DRAM depletion. Therefore, we hypothesize that, downstream of p53, autophagy might sustain inhibition of p53 pro-apoptotic function in a regulatory loop.
Diabetes reduced the effect of chemotherapy that correlated with increased DRAM and reduced PUMA gene expression, in vivo

Finally, the biological effect of the hyperglicemic microenvironment on drug response was evaluated in a nude mouse model of Streptozotocin (STZ)-induced diabetes [33], to recapitulate the in vitro results. After the mice achieved a consistent diabetic state, as evaluated by blood glucose concentration exceeding $300 \mathrm{mg} / \mathrm{dL}$, compared to the normal group that maintained blood glucose concentration around $100 \mathrm{mg} / \mathrm{dL}$ (Fig. 6a), we generated tumor xenografts by injecting RKO cells, as previously reported $[37,38]$, in both diabetic and normoglycemic groups. Cells were implanted into nude mice by i.m. injection and allowed to develop into palpable tumor nodules (about $300 \mathrm{~mm}^{3}$ ) at the injection sites. The mice were then treated with ADR and tumor volumes were calculated from caliper measurements. Ten days after injection only normoglycemic mice treated with ADR displayed significant tumor growth delay (ADR versus Mock: ${ }^{*} P<0.001$ ), compared to the same treatment in diabetic (SZT) mice (Fig. 6b). Then, tumors were harvested and mRNA extracted for p53 target gene expression by RT-PCR analyses. The results show that PUMA was significantly induced by ADR treatment in normoglycemic mice (Fig. 6c) while weakly induced in SZT mice; on the contrary, DRAM was induced by ADR treatment in diabetic mice while was not induced in normoglycemic ones, in agreement with the above in vitro data. This experiment recapitulated the in 

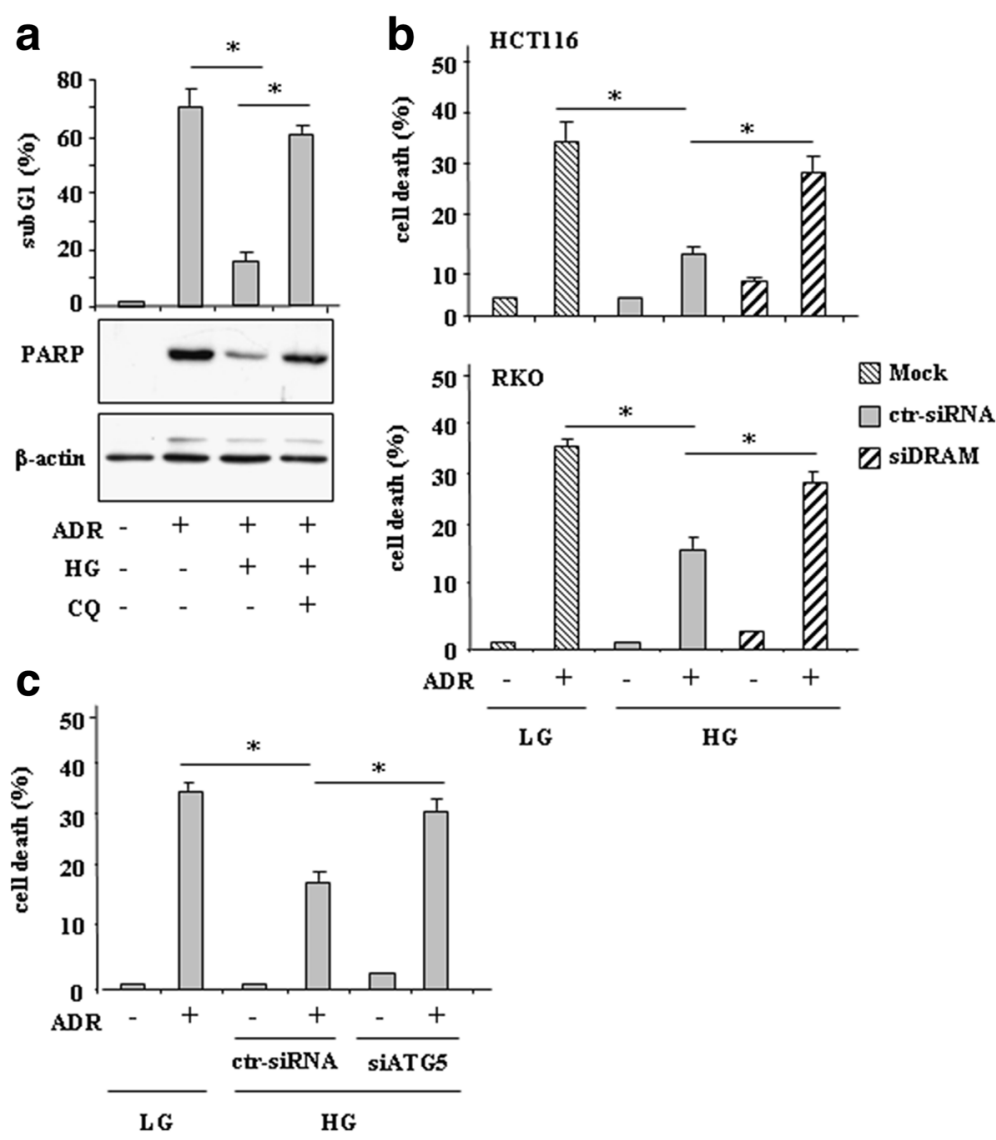

. Autophagy inhibition or DRAM silencing restored ADR-induced cell death in HG. (a) HCT116 cells were treated with ADR (2 $\mu \mathrm{mg} / \mathrm{ml}$ for $24 \mathrm{~h}$ ) in low (-) and high glucose (HG) condition with or without $25 \mu \mathrm{M} C Q$ (for 16 h). After treatments, cells were in part fixed and stained with propidium iodide (PI) for subG1 evaluation (upper panel) or lysed and analyzed by western immunoblotting to assess PARP cleavage (lower panel); relative quantification of PARP cleavage/ $\beta$-actin ratio is shown. One representative experiment is shown. Anti- $\beta$-actin was used as protein loading control. ${ }^{*} P<0.001$. (b) RKO and HCT116 cells, transfected with ctr-siRNA and siDRAM or left untransfected, were kept in low glucose (-) or high glucose (HG) medium for $24 \mathrm{~h}$ and then treated with ADR $(2 \mathrm{\mu g} / \mathrm{ml})$ for $24 \mathrm{~h}$ before the percentage of dead cells was scored by trypan blue exclusion. The data are presented as the means \pm S.D. from three independent experiments. ${ }^{*} P<0.001$. (c) RKO cells, transfected with ctr-siRNA and with siATG5 or left untransfected, were kept in low glucose (-) or high glucose ( $\mathrm{HG}$ ) medium for $24 \mathrm{~h}$ and then treated with ADR $(2 \mathrm{\mu g} / \mathrm{ml})$ for $24 \mathrm{~h}$ before the percentage of dead cells was scored by trypan blue exclusion. The data are presented as the means \pm S.D. from three independent experiments. ${ }^{*} P<0.001$

vitro results and underlined how the diabetic condition might negatively influence tumor response to drugs by impairing p53 pro-apoptotic activity.

\section{Discussion}

PUMA is a potent inducer of apoptosis, whereas inhibiting PUMA curbs apoptosis associated with tissue injury thus inducing therapeutic resistance $[10,11]$. DRAM, by itself or in conjunction with other factors downstream of $\mathrm{p} 53$, can induce autophagy. Intriguingly, DRAM is also involved in p53-induced cell death, although DRAM has no detrimental effect on cell viability when expressed alone $[18,19]$. PUMA and DRAM are two p53 target genes and it is well known that p53 after its activation can select the final biological outcome depending on the triggered transcriptional program [1, 2]. Indeed, although p53 is better known for its proapoptotic activities, it can also induce prosurvival effects, particularly under mild stress conditions. Starting from our previous finding that HG reduces p53 apoptotic activity [25, 26], we found here that the HG condition changed the p53 response upon ADR treatment, driving p53 transcriptional activity from PUMA (death) to DRAM (autophagy) mutual antagonism and that such interplay correlated with impaired druginduced cell death. Despite DRAM has been shown to induce also autophagic cell death, in our system it played a pro-survival role since silencing DRAM restored druginduced cell death inhibited by HG condition. We found that DRAM expression increased the HG-induced autophagy that, in turn, inhibited apoptosis and facilitated the resistance of tumor cells to anticancer agents, in agreement with some studies [22, 23]. Further studies will elucidate whether the PUMA to DRAM differential expression is evident also in tumor 


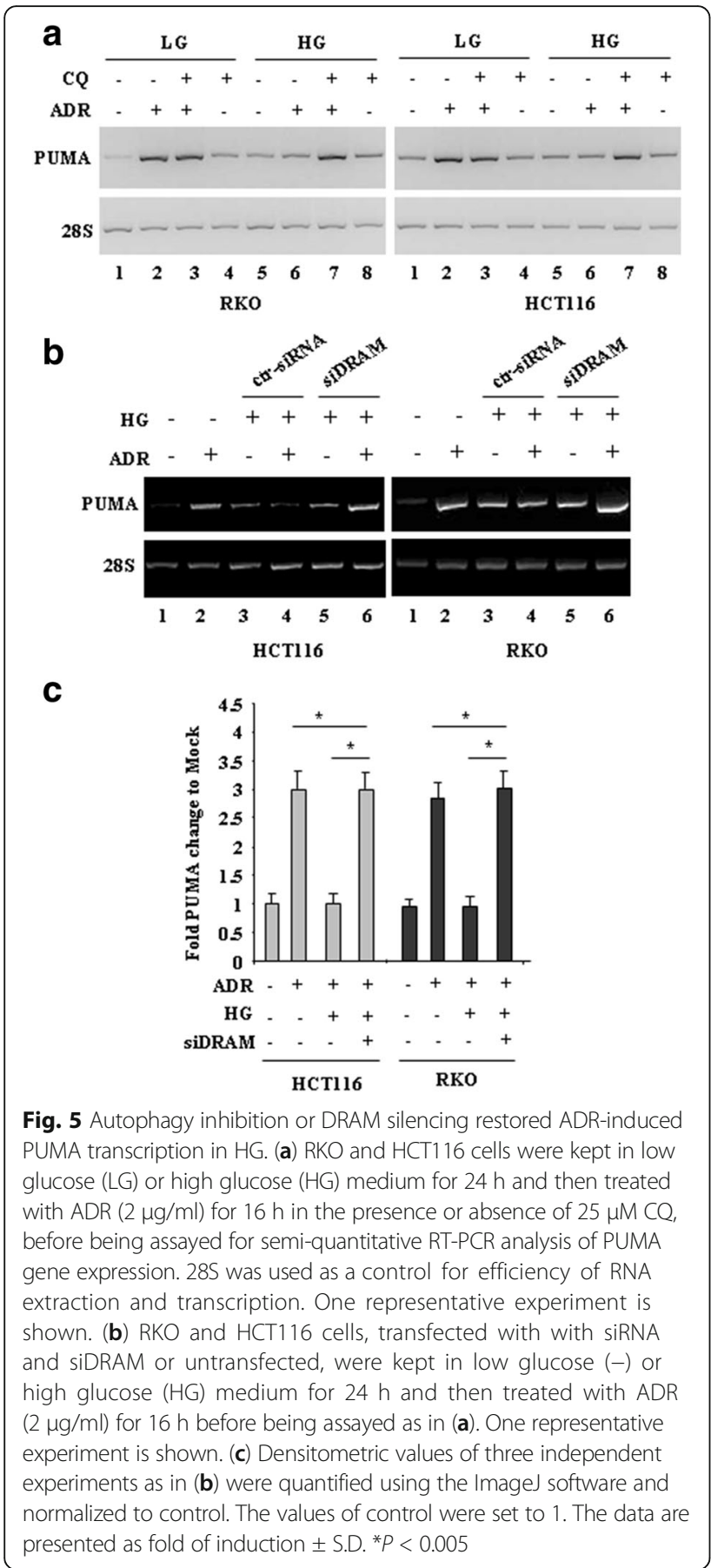

tissues and whether it might be linked to tumor resistance to drugs.

The PUMA to DRAM interplay was the result of p53 activation since blocking p53 transcriptional activity or using p53 null cells abolished such expression. Given that p53 can induce apoptosis in response to high genotoxic stress, it is plausible that HG might modify the way through which p53 selectively induced gene transcription. We know that p53 phosphorylation at serine

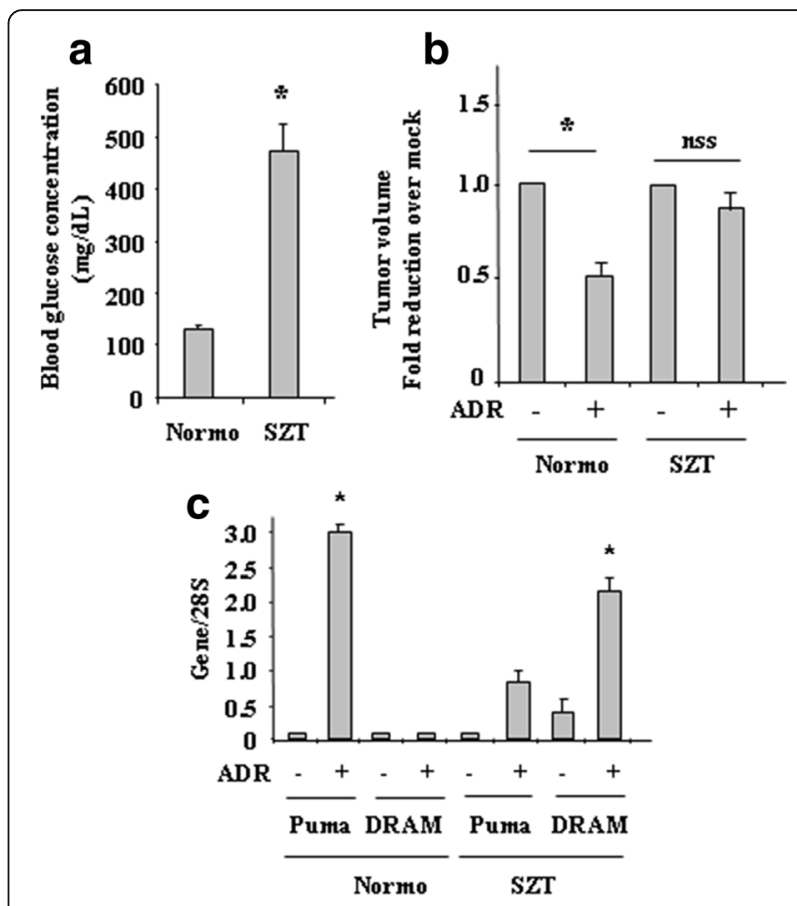

Fig. 6 Diabetes reduced the effect of chemotherapy that correlated with increased DRAM and reduced PUMA gene expression, in vivo. (a) Blood glucose concentration was evaluated in normoglycemic (Normo) and streptozotocin (SZT)-treated mice (diabetic). Data are presented as means \pm S.D. ${ }^{*} P<0.005$. (b) After mice reached a glucose concentration exceeding $300 \mathrm{mg} / \mathrm{dl}$ (considered diabetic) upon SZT treatment, solid tumors were obtained by injecting i.m. RKO cells on the flank of each mouse. ADR treatment was performed when the tumors became palpable. Ten days after ADR treatment, the size of tumors showed statistical significant growth delay in normoglycemic versus SZT mice. The data are presented as fold reduction \pm S.D. ${ }^{*} P<0.005$. (c) Tumors measured in (b) were then explanted from normoglycemic (Normo) and streptozotocin (SZT)-treated mice and total mRNA was analysed by RT-PCR of PUMA and DRAM gene expression. 285 was used as a control for efficiency of RNA extraction and transcription. (c) Densitometric analysis of gene expression in (b) was plotted as expression ratio to $28 \mathrm{~S}$. ${ }^{*} P<0.001$

46 (Ser46) is a critical modification for induction of irreversible apoptosis and that inhibition of such phosphorylation by HG contributes to chemoresistance and reduction of p53-induced apoptosis [39, 40]. We also know that mainly HIPK2 phosphorylates p53Ser46 and that HG triggers HIPK2 degradation, impairing p53 apoptotic response $[25,41]$. In agreement, we found that PUMA expression is related to p53Ser46 modification $[25,26]$. However, although we found here mutual interplay between PUMA to DRAM transcription, whether p53-induced DRAM expression depends on specific p53 modification (i.e., phosphorylation, acetylation, ect.) and/or different extent of genotoxic stress, needs to be clarified.

DRAM is a lysosomal protein and a stress-induced regulator of autophagy that was firstly discovered by microarray analysis of mRNA species responsive to p53 
and associated to autophagy induction [18], although the effect of DRAM-mediated autophagy has not been clarified until now. Starvation has been shown to activate the PI3K/AKT pathway that inhibits apoptosis by DRAMmediated autophagy in hepatocellular cancer cells [42]. We can speculate that the loss of HIPK2/p53Ser46 phosphorylation in high glucose changes the p53 transcriptional activation from pro-apoptotic (PUMA) gene toward autophagy (DRAM) gene. Thus, DRAM silencing restored the ADR-induced cell death in high glucose and inhibited autophagy, highlighting a pro-survival effect of DRAM, likely related to autophagy induction. Whether this effect is cell specific or context-dependent needs further studies.

Autophagy has been shown to have a pro-survival effect in response to chemotherapy when for instance is activated by endoplasmic reticulum stress or by reactivespecies oxygen (ROS) and to induce chemoresistance [23, 43-46]. Our present studies show that blocking autophagy with CQ or by depletion of ATG5 gene, restored the ADR-dependent cell death, highlighting a pro-survival effect of autophagy in this model. Interestingly, blocking autophagy restored PUMA transcription. This was somehow surprisingly. Preliminary findings indicate that HIPK2 might be involved in PUMA to DRAM interplay (data not shown). Further studies will be needed to address whether HIPK2 might be degraded also by autophagy other than by proteasome, as previously reported [47].

\section{Conclusions}

In conclusion, these findings uncover the antagonistic role of PUMA and DRAM to govern drug-induced cell death, highlighting how metabolic conditions such as hyperglicemia might change p53 response to anticancer therapies. These findings might be exploited to design personalized therapies that combine the use of autophagy inhibitors to improve the efficacy of chemotherapy in hyperglicemic patients.

\section{Abbreviations \\ ADR: Adriamycin; CQ: Chloroquine; DMEM: Dulbecco's Modified Eagle's medium; DRAM: Damage-regulated autophagy modulator; FACS: Fluorescence Activated Cell Sorting; FBS: Fetal bovine serum; HBSS: Hanks' Balanced Salt solution; HG: High glucose; LC3: Microtubule-associated protein light chain 3; LG: Low glucose; PI: Propidium iodide; pifithrin-a: PFT-a; PUMA: p53 upregulated modulator of apoptosis; PVDF: polyvinylidene difluoride; RT-PCR: reverse transcription polymerase chain reaction; SD: Standard deviation; siRNA: small-interference RNA; STZ: Streptozotocin}

\section{Acknowledgements}

This study was funded by the Italian Association for Cancer Research (AIRC) Grant to GD (IG 2015 Id.16742) and by Ministry of Health (RF-2011-02347907) to GT. We thank M. Granato, S. Soddu and A. Verdina for sharing reagents and for critical discussion.

\section{Authors'contributions}

GDO designed the study. AG, GP, SB, and GT performed experiments. GDO, and $\mathrm{MC}$ analysed data. GDO wrote the paper. All authors read and approved the final manuscript.

\section{Competing interests}

The authors declare that they have no competing interests.

\section{Publisher's Note}

Springer Nature remains neutral with regard to jurisdictional claims in published maps and institutional affiliations.

\section{Author details \\ 'Department of Research, Advanced Diagnostics, and Technological Innovation, Translational Research Area, Regina Elena National Cancer Institute, Rome, Italy. ${ }^{2}$ Department of Medical, Oral and Biotechnological Sciences, Tumor Biology Section, University "G. d'Annunzio", Via de Vestini, 31, 66013 Chieti, Italy. ${ }^{3}$ Department of Systems Medicine, University "Tor Vergata", Rome, Italy. ${ }^{4}$ Department of Experimental Medicine, Institute Pasteur Cenci Bolognetti Foundation, Sapienza University, Rome, Italy.}

Received: 26 July 2017 Accepted: 5 September 2017 Published online: 11 September 2017

\section{References}

1. Carvajal LA, Manfredi JJ. Another fork in the road-life or death decisions by the tumour suppressor p53. EMBO Rep. 2013;14:414-21.

2. Vousden $\mathrm{KH}$, Prives C. Blinded by the light: the growing complexity of $\mathrm{p} 53$. Cell. 2009:137:413-31.

3. Muller PAJ, Vousden KH. P53 Mutations in cancer. Nat Cell Biol. 2013;15:2-8.

4. Weber JD, Zambetti GP. Renewing the debate over p53 apoptotic response. Cell Death Diff. 2003;10:409-12.

5. Cirone M, Di Renzo L, Lotti LV, Conte V, Trivedi P, Santarelli R, et al. Activation of dendritic cells by tumor cell death. Oncoimmunology. 2012;1:1218-9.

6. Cirone M, Garufi A, Di Renzo L, Granato M, Faggioni A, D'Orazi G. Zinc supplementation is requie for the cytotoxic and immunogenic effects of chemotherapy in chemoresistant p53-functionally deficient cells. Oncoimmunology. 2013;2:e26198.

7. Pistritto G, Trisciuoglio D, Ceci C, Garufi A, D'Orazi G. Apoptosis as anticancer mechanism: function and dysfunction of its modulators and targeted therapeutic strategies. Aging-US. 2016:8:603-19.

8. Nakano K, Vousden KH. PUMA, a novel proapoptotic gene, is induced by p53. Mol Cell. 2001;7:683-94.

9. Yu J, Wang Z, Kinzler KW, Vogelstein B, Zhang L. PUMA mediates the apoptotic response to p53 in colorectal cancer cells. Proc Natl Acad Sci U S A. 2003;100: 1931-6.

10. Hikisz P, Kilianska ZM. PUMA, a critical mediator of cell death - one decade on from its discovery. Cell Mol Biol Lett. 2012:17:646-69.

11. Yu J, Zhang L. PUMA a potent killer with or without p53. Oncogene. 2009; 27:S71-83.

12. Delbridge ARD, Strasser A. The BCL-2 protein family, BH3-mimetics and cancer therapy. Cell Death Diff. 2015;22:1071-80.

13. Rayan KM. p53 And autophagy in cancer: Guardian of the genome meets guardian of the proteasome. Eur J Cancer. 2011:47:44-50.

14. Tasdemir E, Maiuri MC, Galluzzi L, Vitale I, Djavaheri-Mergny M, D'Amelio M, et al. Regulation of autophagy by cytoplasmic p53. Nat Cell Biol. 2008;10:676-87.

15. Scherz-Shouval R, Weidberg H, Gonen C, Wilder Z, Oren M. p53-Dependent regulation of autophagy protein LC3 supports cancer cell survival under prolonged starvation. Proc Natl Acad Sci U S A. 2010:107:18511-6.

16. Kenzelmann-Broz D, Spano Mello S, Bieging KT, Jiang D, Dusek RL, Brady CA, et al. Global genomic profiling reveals an extensive p53-regulated autophagy program contributing to key p53 responses. Genes Dev. 2013;27:1016-31.

17. Jing K, Song KS, Shin S, Kim N, Jeong S, Oh HR, et al. Docosahexaenoic acid induces autophagy through p53/AMPK/mTOR signaling and promotes apoptosis in human cancer cells harboring wild-type p53. Autophagy. 2011:7:1348-58.

18. Crighton D, Wilkinson S, O'Prey J, Syed N, Smith P, Harrison PR, et al. DRAM, a p53- induced modulator of autophagy, is critical for apoptosis. Cell. 2006; 126:121-34.

19. Crighton D, Wilkinson S, Ryan KM. DRAM links autophagy to $\mathrm{p} 53$ programmed cell death. Autophagy. 2007;3:72-4. 
20. Maiuri MC, Galluzzi L, Morselli E, Kepp O, Malik SA, Kroemer G. Autophagy regulation by p53. Curr Opin Cell Biol. 2010;22:181-5.

21. Green DR, Levine B. To be or not to be? How selective autophagy and cell death govern cell fate. Cell. 2014;157:65-75.

22. Boya P, Gonzalez-Polo RA, Casares N, Perfettini JL, Dessen P, Larochette N, et al. Inhibition of macroautophagy triggers apoptosis. Mol Cell Biol. 2005; 25:1025.1040.

23. Huang Z, Zhou L, Chen Z, Nice EC, Huang C. Stress management by autophagy: implications for chemoresistance. Int J Cancer. 2016;139:23-32.

24. Marino G, Niso-Santano M, Baehrecke EH, Kroemer G. Self consumption: the interplay of autophagy and apoptosis. Nat Rev Mol Cell Biol. 2014;15:81-94.

25. Garufi A, D'Orazi G. High glucose dephosphorylates serine 46 and inhibits p53 apoptotic activity. J Exp Clin Cancer Res. 2014;33:79.

26. Baldari S, Garufi A, Granato M, Pistritto G, Cirone M, D'Orazi G. Hyperglycemia triggers HIPK2 protein degradation. Oncotarget. 2017;8:1190-203.

27. Biernacka KM, Uzoh CC, Zeng L, Persad RA, Bahl A, Gillatt D, et al. Hyperglicaemiainduced chemoresistance of prostate cancer cells due to IGFBP2. Endocr Relat Cancer. 2013;20:741-51.

28. Ma YS, Yang IP, Tsai HL, Huang CW, Juo SH, Wang JY. High glucose modulates antiproliferative effect and citotoxicity of 5-fluorouracil in human colon cancer cells. DNA Cell Biol. 2014:33:64-72.

29. Michihara A, Toda K, Kubo T, Fujiwara Y, Akasaki K, Tsuji H. Disruptive effect of choroquine on lysosomes in cultured rat hepatocytes. Biol Pharm Bull. 2005;28:947-51.

30. Komarov PG, Komarova EA, Kondratov RV, Christov-Tselkov K, Coon JS, Chernov MV, et al. A chemical inhibitor of p53 that protects mice from the side effects of cancer therapy. Science. 1999;285:1733-7.

31. Garufi A, Trisciuoglio D, Porru M, Leonetti C, Stoppacciaro A, D'Orazi V, et al. A fluorescent curcumin-based $\mathrm{Zn}(\mathrm{II})$-complex reactivates mutant $(\mathrm{R} 175 \mathrm{H}$ and R273H) p53 in cancer cells. J Exp Clin Cancer Res. 2013;32:72.

32. Garufi A, Ricci A, lorio E, Carpinelli G, Pistritto G, Cirone M, et al. Glucose restriction induces cell death in parental but not in HIPK2 depleted RKO colon cancer cells: molecular mechanisms and implications for tumor therapy. Cell Death Disease. 2013;4:e639.

33. Graham ML, Janecek JL, Kittredge JA, Hering BJ, Schuurman HJ. The streptozotocininduced diabetic nude mouse model: differences between animals from different sources. Comp Med. 2011;61:356-60.

34. Crighton D, O'Perey J, Bell HS, Ryan KM. p73 Regulates DRAM-independent autophagy that does not contribute to programmed cell death. Cell Death Diff. 2007;14:1071-9.

35. Klionsky DJ, Abdelmohsen K, Abe A, Abedin MJ, Hagai Abeliovich H, Arozena AA, et al. Guidelines for the use and interpretation of assays for monitoring autophagy (2nd edition). Autophagy. 2016;12:1-222.

36. Mizushima N, Yoshimori T, Ohsumi Y. The role of Atg proteins in autophagosome formation. Annu Rev Cell Dev Biol. 2011;27:107-32.

37. Puca R, Nardinocchi L, Gal H, Rechavi G, Amariglio N, Domany E, et al. Reversible dysfunction of wild-type p53 following homeodomain-interacting protein kinase2 knockdown. Cancer Res. 2008;15:3707-14.

38. Garufi A, Ubertini V, Mancini F, D'Orazi V, Baldari S, Moretti F, et al. The beneficial effect of zinc(II) on low-dose chemotherapeutic sensitivity involves p53 activation in wild-type p53 cancer cells. J Exp Clin Cancer Res. 2015;34:87.

39. Oda K, Arakawa H, Tanaka T, Matsuda K, Tanikawa C, Mori T, et al. P53AIP1, A potential mediator of p53-dependent apoptosis, and its regulation by Ser-46-phosphorylated p53. Cell. 2000;102:849-62.

40. Ichwan SJA, Yamada S, Sumreijkanchanakii P, Ibrahim-Auerkari E, Eto K, Ikeda MA Defect in serine 46 phosphorylation of p53 contributes to acquisition of p53 resistance in oral squamous cell carcinoma cells. Oncogene. 2006;25:1216-24.

41. D'Orazi G, Cecchinelli B, Bruno T, Manni I, Higashimoto Y, Saito S, et al. Homeodomain-interacting protein kinase-2 phosphorylates p53 at Ser46 and mediates apoptosis. Nat Cell Biol. 2002;4:11-9.

42. Liu K, Shi Y, Guo XH, Ouyang YB, Wang SS, Liu DJ, et al. Phoaphorylated AKT inhibits the apoptosis induced by DRAM-mediated mitophagy in hepatocellular carcinoma by preventing the translocation of DRAM to mitochondria. Cell Death Dis. 2014:5:e1078

43. Granato M, Santarelli R, Lotti LV, Di Renzo L, Gonnella R, Garufi A, et al. JNK and macroautophagy activation by bortezomib has a pro-survival effect in primary effusion lymphoma cells. PLoS One. 2013;8:e75965.

44. Yan YL, Xu ZJ, Dai S, Qian L, Sun LQ, Gong ZC. Targeting autophagy to sensitive glioma to temozolomide treatment. J Exp Clin Cancer Res. 2016;35:23.

45. Panieri E, Santoro MM. ROS homeostasis and metabolism: a dangerous liaison in cancer cells. Cell Death Dis. 2016;7:e2253.
46. Galadari S, Rahman A, Pallichankandy S, Thayyullathil F. Reactive oxygen species and cancer paradox: to promore or to suppress? Free Radic Biol Med. 2017;104:144-64

47. Nardinocchi L, Puca R, Givol D, D'Orazi G. HIPK2-A therapeutical target to be (re)activated for tumor suppression: role in p53 activation and HIF-1alpha inhibition. Cell Cycle. 2010;9:1-6.

\section{Submit your next manuscript to BioMed Central and we will help you at every step:}

- We accept pre-submission inquiries

- Our selector tool helps you to find the most relevant journal

- We provide round the clock customer support

- Convenient online submission

- Thorough peer review

- Inclusion in PubMed and all major indexing services

- Maximum visibility for your research

Submit your manuscript at www.biomedcentral.com/submit

) Biomed Central 